\title{
Plasmonic cloaking for irregular objects with anisotropic scattering properties
}

\author{
S. Tricarico, ${ }^{1}$ F. Bilotti, ${ }^{1, *}$ A. Alù, ${ }^{2, \dagger}$ and L. Vegni ${ }^{1}$ \\ ${ }^{1}$ Department of Applied Electronics, University "Roma Tre," Via della Vasca Navale, 84-00146 Rome, Italy \\ ${ }^{2}$ Department of Electrical and Computer Engineering, The University of Texas-Austin, 1 University Station, \\ C0803 Austin, Texas 78712, USA
}

(Received 29 October 2009; published 18 February 2010)

\begin{abstract}
Here we extend the plasmonic cloaking technique to irregularly shaped objects with anisotropic scattering response. The scattering-cancellation approach to cloaking [A. Alù and N. Engheta, Phys. Rev. E 72, 016623 (2005)] has been extensively applied in the past to symmetrical geometries and canonical shapes. However, recent papers have raised some doubts concerning the fact that its use may not be as effective when dealing with strongly anisotropic and noncanonical geometries. Our goal here is to extend the plasmonic cloaking technique to irregular obstacles and to show that proper cloak design may provide a significant and uniform scattering reduction, independent of angle of incidence, position, and polarization of the illumination. We investigate how the volumetric effect of scattering cancellation provided by plasmonic media may drastically suppress the scattering for these irregular geometries independent of the illumination angle, and we shed some light on the physical mechanisms and the design rules at the basis of this cloaking technique when applied to objects whose scattering properties are dependent upon polarization and angle of incidence.
\end{abstract}

DOI: 10.1103/PhysRevE.81.026602

PACS number(s): 78.67.Pt

\section{INTRODUCTION}

In the last few years, the idea of synthesizing a cloak of invisibility capable of hiding a given object to an external observer has been extensively investigated by several groups worldwide [1-25]. This topic is of great interest for the scientific community, especially for the technological impact that such devices may have in various fields, including telecommunications, optics, or biological sensing. Different physical mechanisms may produce significant scattering reduction, such as coordinate transformations [1-5], scattering cancellation [6-15], anomalous localized resonances [16,17], and transmission-line cloaking networks [18]. Among these, scattering cancellation has shown to be a simple and robust way to design cloaking devices for moderately sized objects. As previously shown [6], by coating a moderately sized object with plasmonic media or metamaterials whose relative electric permittivity may assume values smaller than unity, the overall scattered fields from the object may be dramatically reduced. This implies that the amplitude of the total scattering cross-section (SCS) may be minimized, making the object substantially undistinguishable from the background in a given frequency range. Plasmonic covers indeed exhibit a local negative polarizability, which is able to compensate, and eventually suppress, the overall scattering from the object they cover. This phenomenon is quite intuitive in the Rayleigh limit, when the obstacle scattering is dominated by its dipolar contribution [6], but it may be also extended to the case of relatively larger objects, considering the contribution associated to the higher-order multipolar scattering $[7,8]$.

As analyzed in [9], the scattering cancellation technique relies on the use of simple homogeneous covers against, for

\footnotetext{
*FAX: +39.06.57337026; bilotti@uniroma3.it

†FAX: + 1-512-4716598; alu@ mail.utexas.edu
}

instance, the transformation-based approach that exploits inhomogeneous and anisotropic materials, thus facing critical issues concerning the design and technological manufacturing of the device (especially in the optical regime). Moreover, the scattering cancellation approach is intrinsically nonresonant, providing good performances in terms of operating bandwidth, and showing an undoubted robustness to geometrical or electric variations of the coated object. Metamaterial plasmonic shells may be easily synthesized both at microwaves $[10,11]$ and in the optical regime [12-15], and they may be straightforwardly used to conformably cover the obstacle of interest. The analytical formulations used to design plasmonic cloaks, however, have always assumed in the literature, for simplicity, that the object to be cloaked has a canonical and symmetrical geometry. On the other hand, transformation-based cloaking has seen a relevant number of extensions to arbitrarily shaped objects and cloaks [19-24]. This has generated some confusion in the recent literature, with papers on the topic claiming that the use of a spherical plasmonic cloak may not be successfully employed to hide an irregular or anisotropic shape. The inherent nature of the plasmonic cloaking technique, which is based on a scattering cancellation mechanism, obviously requires that, in order to suppress the scattering response of an anisotropic object, simultaneously at all different angles and polarizations, the cloak should be appropriately designed with an asymmetric and anisotropic shape, which was not considered, for instance, in [25].

Here, we approach this problem in general, and we show that it is indeed possible to envision plasmonic cloaks capable of suppressing the scattering of irregular and anisotropic shapes by properly optimizing not only its plasmonic properties but also its shape and design. In the next sections, we will investigate the performances of such plasmonic covers to operate at optical frequencies, both for regular shapes and for structures characterized by a strongly polarizationdependent anisotropic response to the illuminating electromagnetic field. 

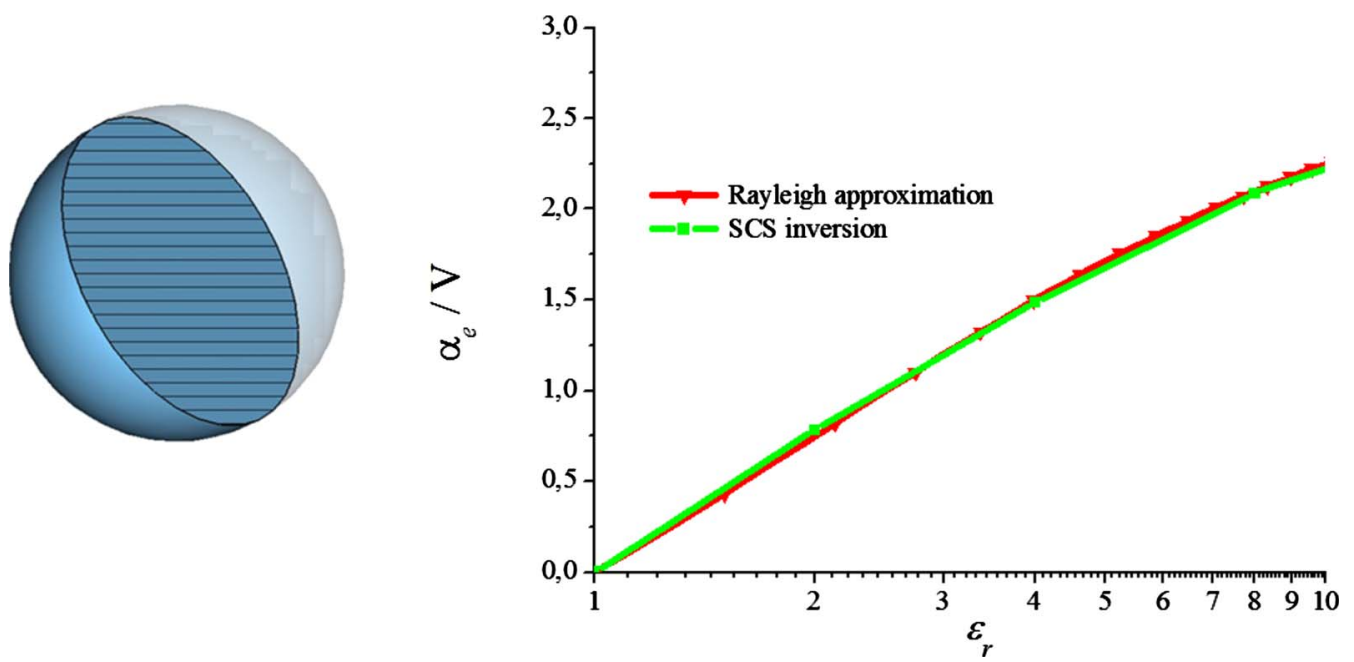

FIG. 1. (Color online) Retrieved normalized electric polarizability $\alpha_{e}$ of a sphere as a function of its relative permittivity.

\section{CLOAKING OF POLYHEDRAL OBJECTS OF MODERATE ELECTRICAL SIZE}

In the Rayleigh approximation (electrically small objects), the scattering cancellation approach is associated with the suppression of the overall dipole moment by inducing a negative dipole moment in the cloak, which may compensate and cancel the scattering from the object [6]. As shown in $[7,8]$, this approach is still valid for relatively larger objects for which the Rayleigh approximation does not apply since the same approach may be used to cancel any dominant multipolar scattering order. When dealing with complex geometries and anisotropic scattering response, as shown in the following, the cover may still be designed to obtain an analogous behavior at the desired frequency, independent of the angle of incidence and polarization of the impinging fields. In fact, since this effect is volumetric [6], it is expected that even irregular shapes may be cloaked as long as the response of the cloak is tailored to provide the proper (anisotropic) polarization effects.

In the long wavelength limit, a generally shaped object with anisotropic scattering properties may be compactly described by a polarizability tensor $\underline{\boldsymbol{\alpha}}[26,27]$ that relates the induced dipole moment vector to the impinging electric field vector: $\mathbf{p}=\varepsilon_{0} \underline{\boldsymbol{\alpha}} \cdot \mathbf{E}_{0}$. It is worth noticing that for many shapes of small scatterers, the polarizability tensor may be assumed to be uniaxial in the proper reference system, ensuring that, although the amplitude of the induced dipole moment may vary with the polarization of the local electric field, the induced dipole moment may be parallel to the electric field when lying along the three main axes of the scatterer. Under this assumption, it may be possible to define a scalar polarizability that depends on the polarization of the electric field along these three main directions.
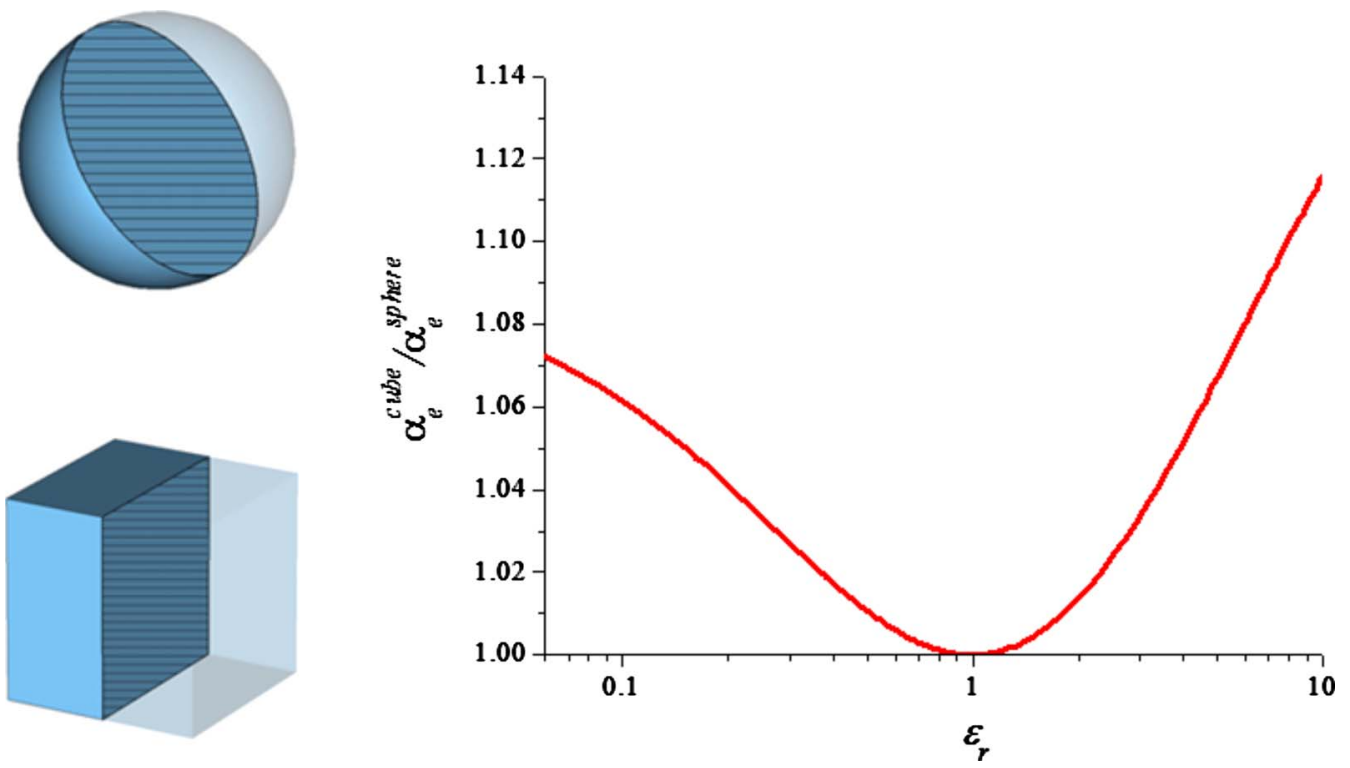

FIG. 2. (Color online) Ratio between the electric polarizabilities of a sphere and the one of a cube (with same volumes) as a function of their relative permittivity. 


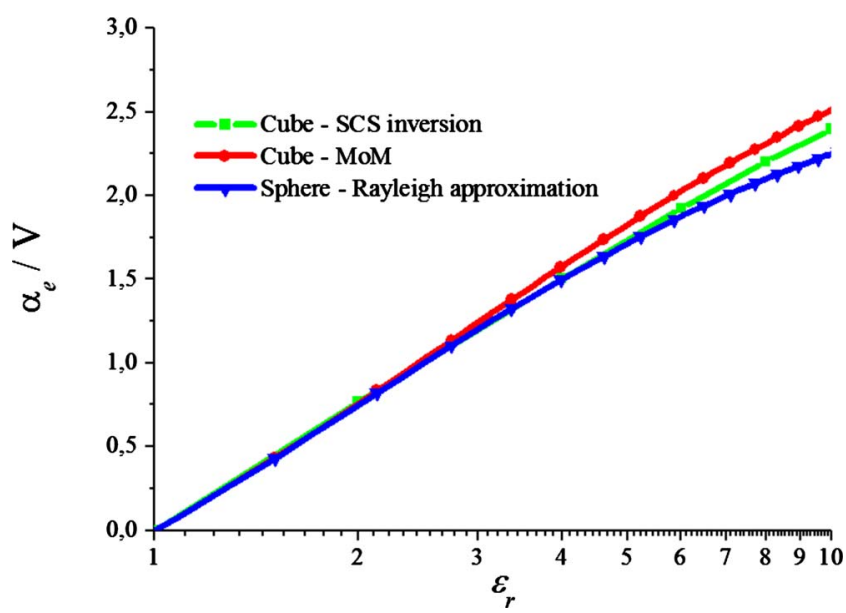

FIG. 3. (Color online) Electric polarizabilities for a cube (orthogonal incidence to one of the faces) and a sphere as a function of their relative permittivity.

Although the scattering from an irregular shape may not always be calculated analytically, we may easily relate its total scattering cross section, evaluated with numerical techniques, to the components of its polarizability tensor for three orthogonal illuminations. This would represent a complete description of the scattering properties of the object of interest as long as its scattering is dominated by the dipolar fields. In this limit, it is easily found that

$$
\alpha_{x x}=\frac{\sqrt{4 \pi \sigma_{x x}}}{k_{0}^{2}},
$$

where $\alpha_{x x}$ is for example the polarizability component for electric field polarized along $\hat{x}, \sigma_{x x}$ is the total scattering cross section for the same polarization and $k_{0}$ is the background wave number. By choosing three orthogonal polarizations of the impinging electric field, we can calculate numerically the independent elements of the polarizability tensor $\underline{\boldsymbol{\alpha}}$ for a given object.

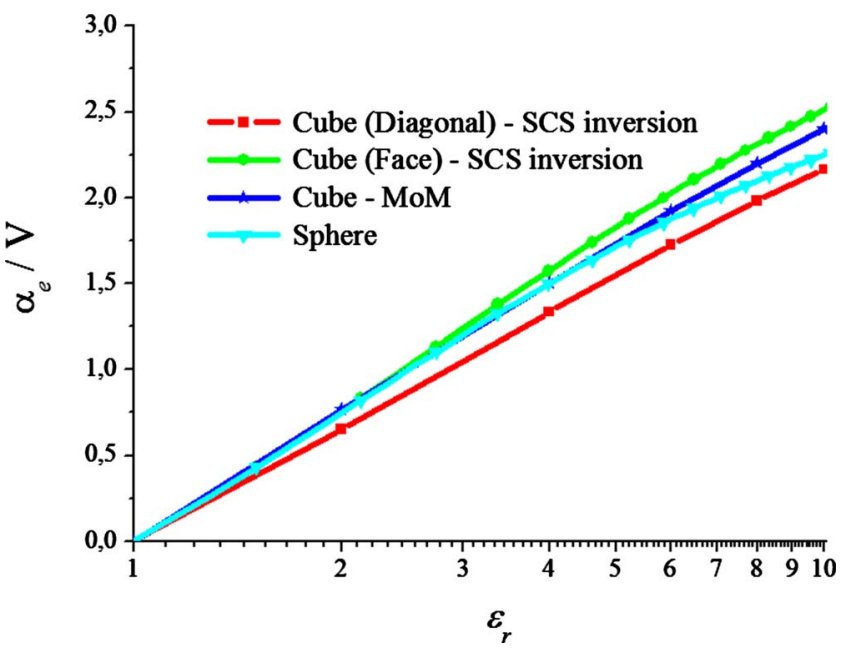

FIG. 4. (Color online) Electric polarizabilities for a cube and a sphere as a function of their relative permittivity.

In order to validate this approach, we have first calculated the far-zone scattering width of an electrically small dielectric sphere of radius $a$ using a commercially available fullwave simulator based on the finite integration technique [28]. In this case, given the spherical symmetry of the particle, the electric polarizability $\alpha_{e}$ is independent from the polarization of the electric field, so the scattering matrix reduces to the scalar $\sigma_{s}$. Then, we numerically integrated the calculated scattering width to compute $\sigma_{s}$, and we compared the polarizability obtained with Eq. (1) to the well known exact solution [29]

$$
\begin{gathered}
\alpha_{e}^{\text {sphere }}=3 V \frac{\varepsilon_{r}-1}{\varepsilon_{r}+2} \\
\sigma_{s}^{\text {sphere }}=4 \pi k_{0}^{4} a^{6}\left(\frac{\varepsilon_{r}-1}{\varepsilon_{r}+2}\right)^{2},
\end{gathered}
$$

in the limit $a \ll \lambda_{0}$ for a volume $V=4 \pi a^{3} / 3$. As shown in Fig. 1 , the retrieved numerical values of $\alpha_{e}$ match the expected solution.
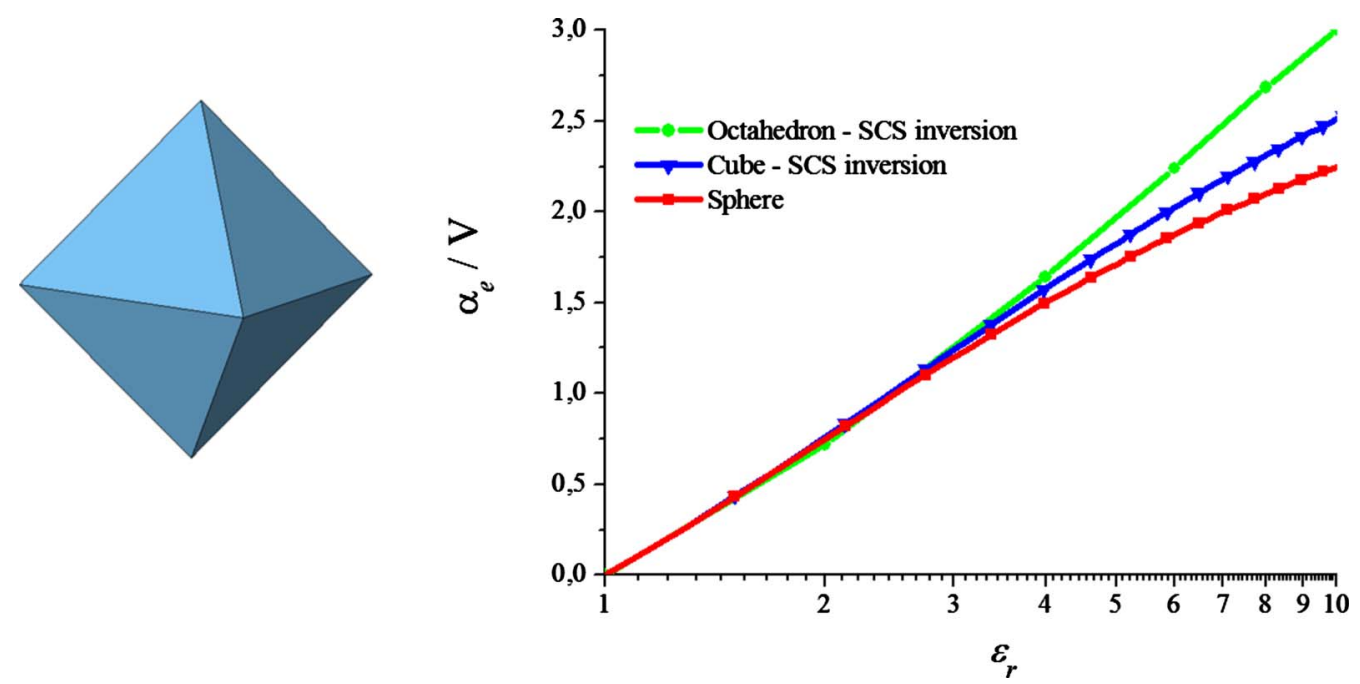

FIG. 5. (Color online) Electric polarizability for an octahedron as a function of their relative permittivity. 

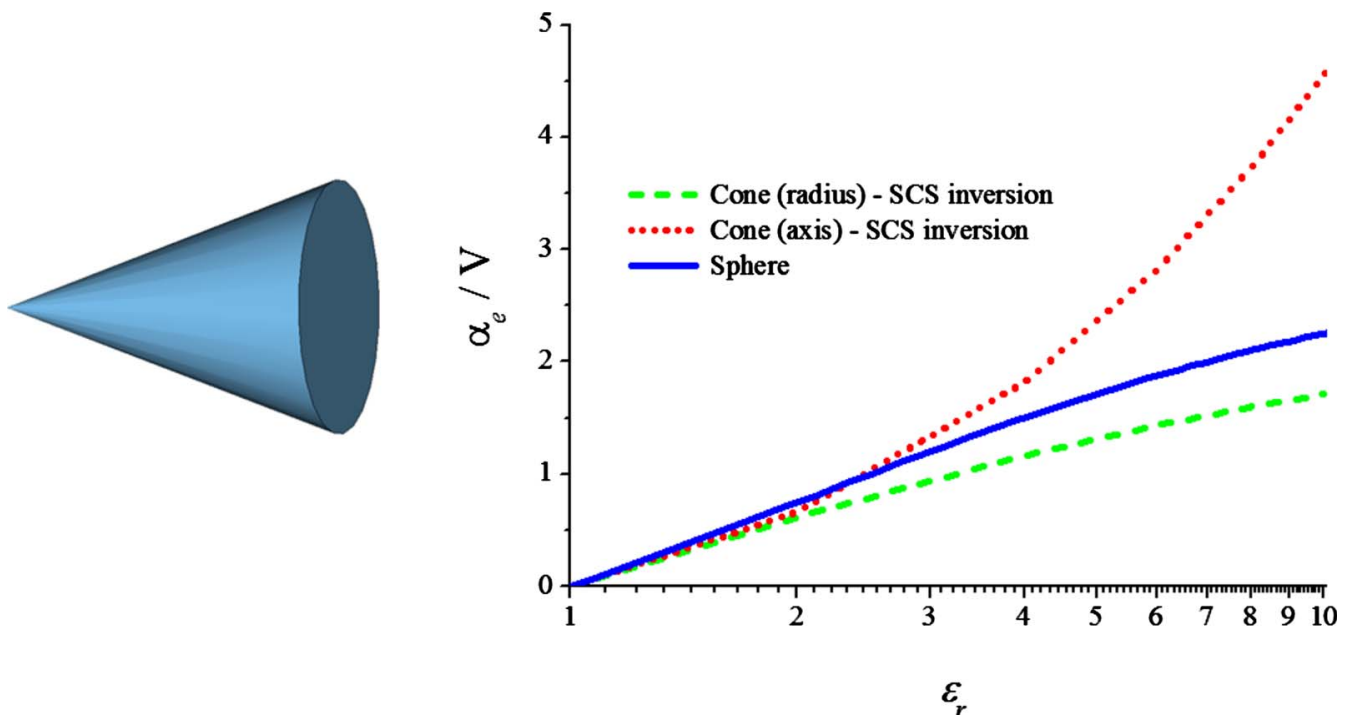

FIG. 6. (Color online) Electric polarizability for a cone as a function of its relative permittivity: impinging electric field directed along the axis (dotted red line), impinging electric field directed along the base radius (dashed green line).

As suggested in [27], if we consider more complex shapes, such as regular polyhedral scatterers, it is easy to find that, due to their high symmetric spatial arrangements of faces, there is no substantially preferred direction, and thus their polarizabilities reduce to scalar quantities as well [27,30,31]. Moreover, it is expected that for small dielectric contrasts the polarizability of these shapes should not sensibly differ from the one of a sphere with the same volume. Similar arguments justify the fact that the main factor in designing the proper plasmonic cloak is that its electrical volume may compensate the one of the object to produce an overall zero polarizability for the composite structure. In Fig. 2 , we show the ratio between the electric polarizabilities of a cube and a sphere with same volume, as a function of their relative permittivity, derived by applying the method of moments (MoMs), as reported in [27,31]. It is evident that, due to the similar values of polarizability in the limit of electrically small particles, a plasmonic cover properly working for a sphere can be also used to cloak a cube, generally working also for different incidence angles. This is consistent with some of the results reported in [25].

In order to extend these results to more asymmetric shapes, we first compare in Fig. 3 the values of $\alpha_{e}$ derived by applying the MoM and SCS inversion for a cubic particle in the case of orthogonal incidence to one of the faces. In Fig. 4 , we consider the case of oblique incidence with impinging wave vector $\hat{\boldsymbol{k}}_{i}$ lying along the diagonal of a cubic face (worst case scenario).

As expected, the cube does not exhibit a strong anisotropic response, but the interesting point is that similar results may be obtained also for a more complex polyhedral shape, such as an octahedron. In this case, as reported in Fig. 5 , the derived values of polarizability are very close to the ones obtained for a an isovolumetric cube.

If we consider now a cone, we expect a substantially different response by changing the propagation direction of the impinging radiation. As shown in Fig. 6, for weak scatterers (i.e., small dielectric contrasts), the electric polarizability is still comparable with the one of a bare sphere, but increasing the relative permittivity of the dielectric, the anisotropic behavior indeed may not be neglected.

An immediate consequence of the previous results is that we may be able not only to design a proper cover to cloak electrically small objects, but we may use a shell designed for a spherical geometry to properly work also for a different shape, as long as the electrical size of the object is small enough to ensure quasiuniform field in the object. For larger objects, however, the situation is different and a wiser cloak design should be employed to make sure that the different polarizability of an anisotropic object for different field polarization is properly taken into account.

Let us consider a spherical silver particle of radius $R$ of permittivity $\varepsilon$ surrounded by a plasmonic cover with radius $R_{c}$ (see Fig. 7). As shown in [6-9], we can easily find the relative permittivity of the cover to sensibly reduce the SCS of the structure. Assuming, for example, $R=50 \mathrm{~nm}$ and $R_{c}$ $=1.8 R$, we get for the external shell permittivity $\varepsilon_{r} \approx 0.5$ at $600 \mathrm{THz}$. Now, let us explore the possibility to cloak a cubic object. It is obvious that in the general case we cannot simply put a smaller cubic shape fitting the spherical core inside the cloak, as suggested in [25]. Following the previous analysis, in the quasistatic limit the same cloak may work only for a cube having similar physical volume as the sphere.

In order to clarify this point, let us consider the asymptotic behavior of scattering coefficients in the quasistatic limit for a coated electrically small spherical object.
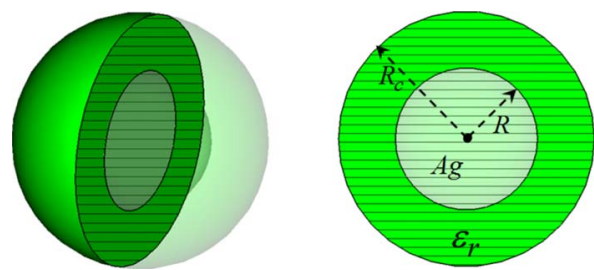

FIG. 7. (Color online) A silver sphere covered with a plasmonic shell. 


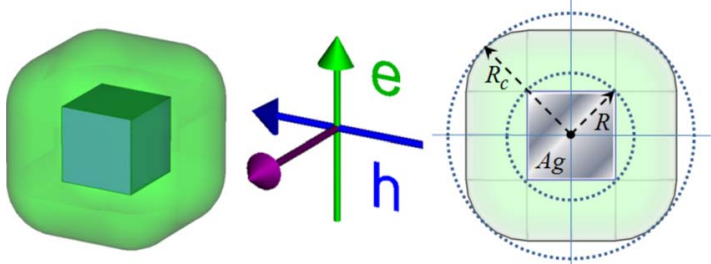

FIG. 8. (Color online) A conformal plasmonic cover for a silver cube.

Considering only the dipolar term in the Mie expansion [6] the total dipole moment of the overall system (core+cover) is suppressed if the following relation holds:

$$
V(\varepsilon-1)+\frac{V_{c}}{3}\left(\varepsilon_{r}-1\right)\left(\frac{\varepsilon}{\varepsilon_{r}}+2\right)=0,
$$

where $V$ and $V_{c}$ are the volumes of the inner particle and of the shell, respectively. Expression (3) is formally equivalent to the one derived for arbitrarily shaped cylindrical objects in [10] for the two-dimensional (2D) case, introducing a condition on the ratio between the involved volumes and their dielectric contrasts, which is directly associated with the overall polarizability of the object and cloak (2), consistent with the results reported in [6]. Since the transparency condition is based on a nonresonant volumetric phenomenon of cancellation of the induced electric dipole moments, following the previous results, it is expected that this same condition may be applied to nonspherical objects.

Consider for instance a cubic scatterer, as in Fig. 8. Proper cloaking may be achieved by deforming the cloak shape to adhere conformably to the cubic surface, keeping the same filling factor as in Eq. (3), but preserving the shape of the hidden object, as depicted in Fig. 8. This may ensure that, independent of the angle of incidence, the overall polariz-
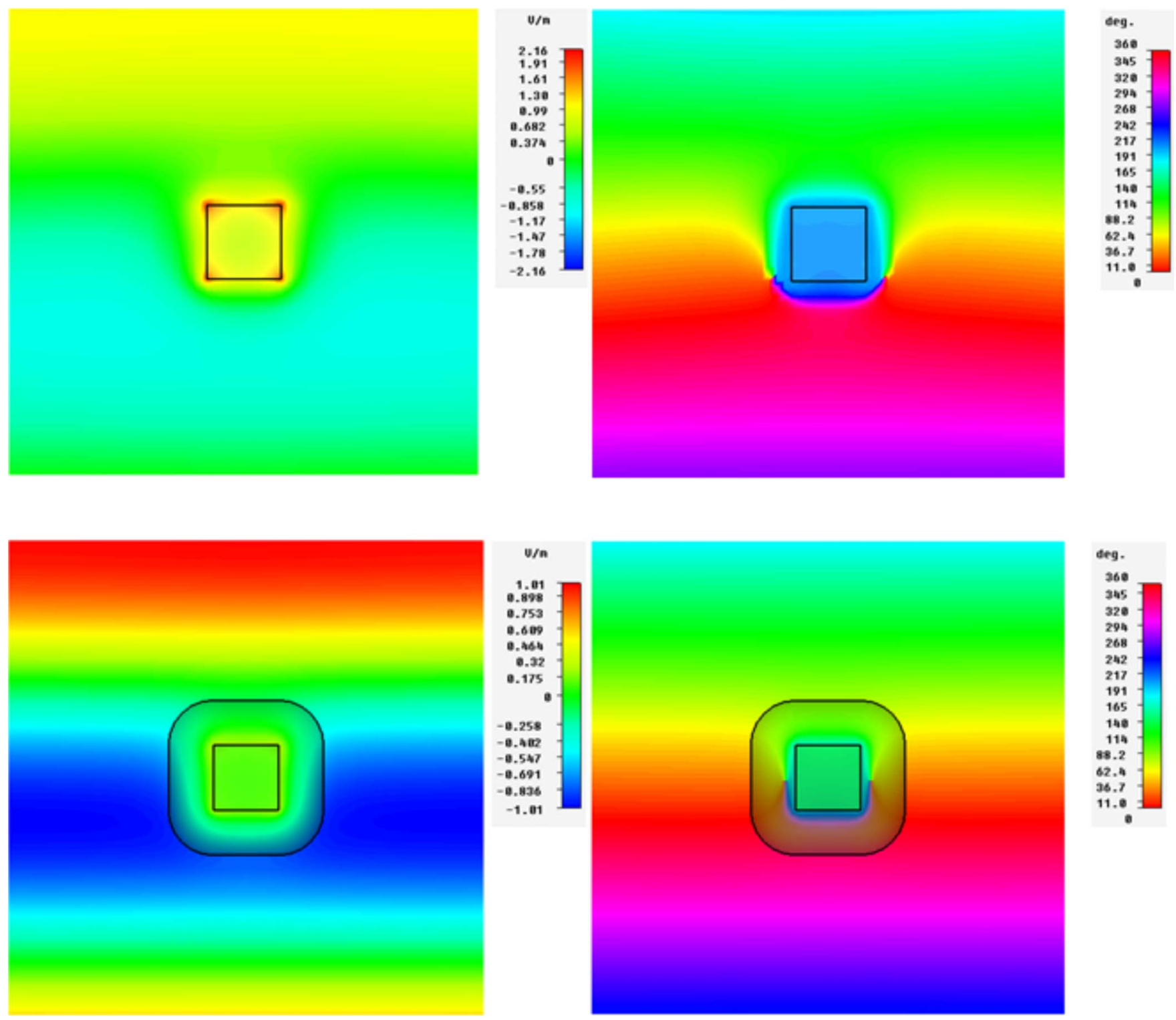

FIG. 9. (Color online) 2D maps of the electric field amplitude (left) and phase (right) for the bare silver cube (top) and the cloaked one (bottom) for plane wave incidence from bottom to top with incident electric field of $1 \mathrm{~V} / \mathrm{m}$. 


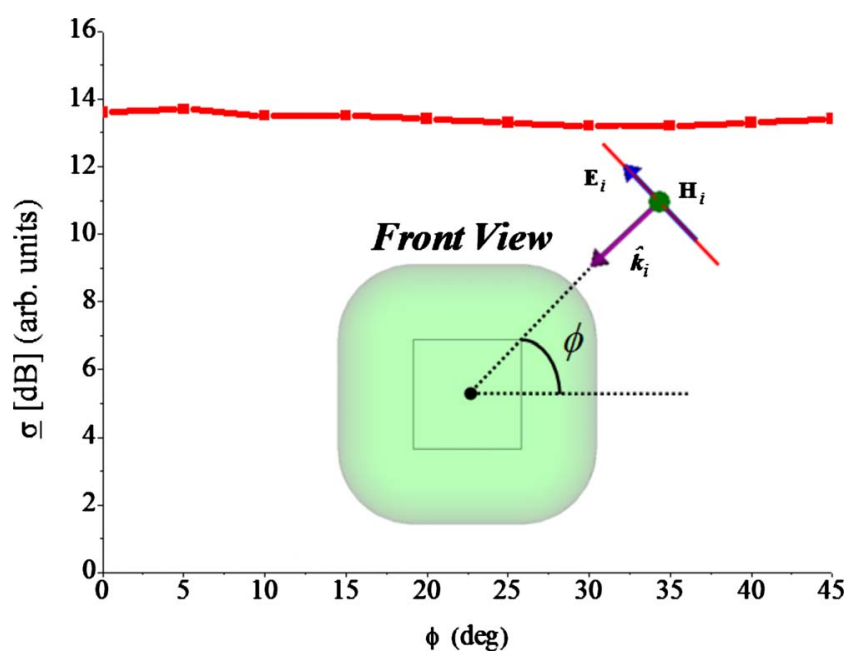

FIG. 10. (Color online) Total scattering reduction between the SCS of the bare cube and the cloaked one as a function of the incidence angle.

ability of the cube and the cloak are opposite to each other.

Following these considerations, we have simulated a cloaked cube with same value of permittivity as in the spherical setup. The silver was modeled as a Drude dispersive material with losses [32]. As expected, we obtain similar performances at the design frequencies, resulting in a total scattering reduction of almost $14 \mathrm{~dB}$ with respect to the case of the bare cube. In Fig. 9, we have reported the 2D field maps, both in amplitude and phase, for the electric and magnetic field distribution. We have assumed here plane wave incidence from bottom to top with an electric field amplitude of $1 \mathrm{~V} / \mathrm{m}$. It is noticed that the field is identically restored with proper cloaking, both in amplitude and phase, and the electric field enhancement at the cube vertices, which is mainly responsible for the scattering in this case, are eliminated by the presence of the cloak.

In Fig. 10, we report the variation in the total scattering reduction $(\sigma)$, that is, the ratio between the SCS of the bare object and the cloaked one, with the angle of incidence of the illuminating plane wave, varying from incidence orthogonal to one face to the case of incidence on the cube edge. It is evident that the conformal design of the cloak ensures simi-
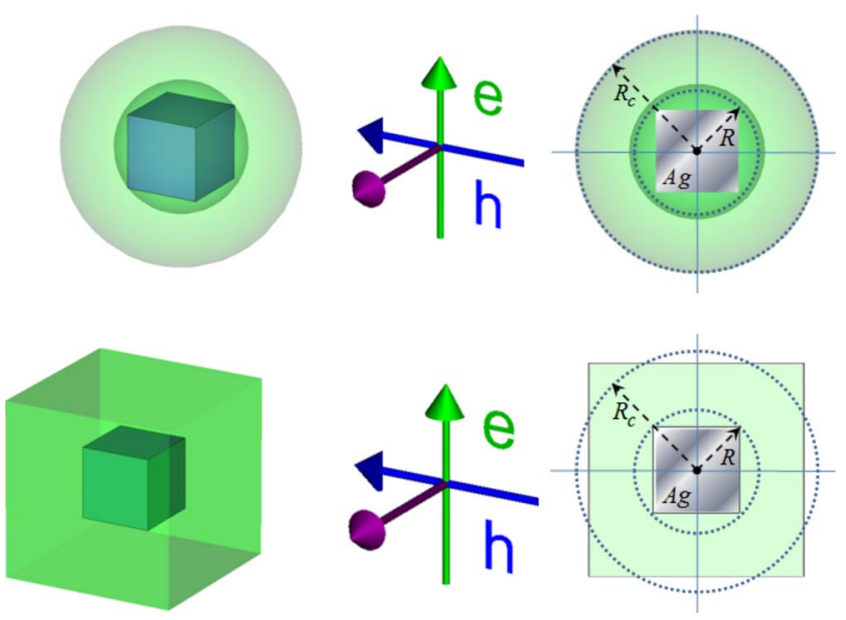

FIG. 11. (Color online) A silver cubic particle surrounded by a plasmonic spherical shell (top) and by a cubic one (bottom).

lar cloaking performance independent of the angle of incidence (Fig. 11).

We consider now two different geometries for the cloak, keeping the same cubic object: the use of the original spherical shape and the use of a cubical shape without rounded corners.

In these cases the scattering reduction is worsened, as expectable, even though the spherical cloak still may achieve a reasonable scattering reduction using high filling ratios. In this case, however, the scattering reduction is more anisotropic due to the difference in effective polarizability when impinging at an edge of the cube, as shown in Fig. 12 (right panel). In the cubic cloak case (left panel), the change in effective volume of the cloak and the presence of sharp edges and vertices produce additional scattering, that worsens the overall performance compared to Fig. 10.

The previous examples show that for moderately anisotropic objects it may indeed be possible to design a cloaking plasmonic layer effective over a broad range of angles and polarization, and with quasi-isotropic response. The best solution is to design a conformal cloak with rounded shapes conformal to the object of interest, which provides similar drastic scattering cancellation performance for all angles of incidence. The cloak may still keep its simplicity of design,
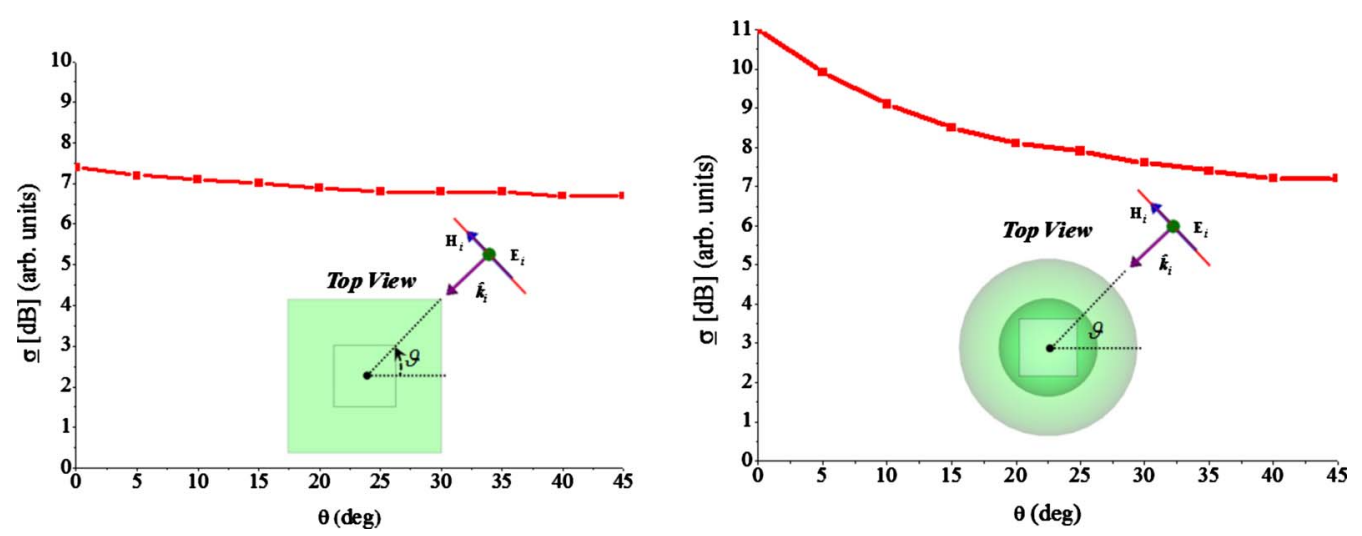

FIG. 12. (Color online) Ratio between the SCS of the bare cube and the cloaked one as a function of the incidence angle for a particle surrounded by a nonrounded cubic shell (left) and by a spherical one (right). 

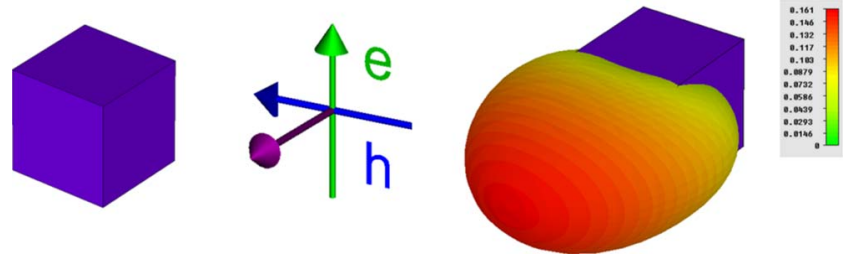

FIG. 13. (Color online) Radar cross-section of a dielectric cube of silica for the given polarization.

relying on homogeneous and isotropic materials, but the thickness of the cloak should be locally chosen to conform to the local variation in the shape of the object. When the geometry gets electrically larger and/or more anisotropic, simple volumetric formulation (3) is not strictly applicable since the ideal assumption of quasistatic fields in the object and the cloak is not valid. Also in this case, however, a proper design of the cloak using physical considerations may be carried out, as shown in the next Section.
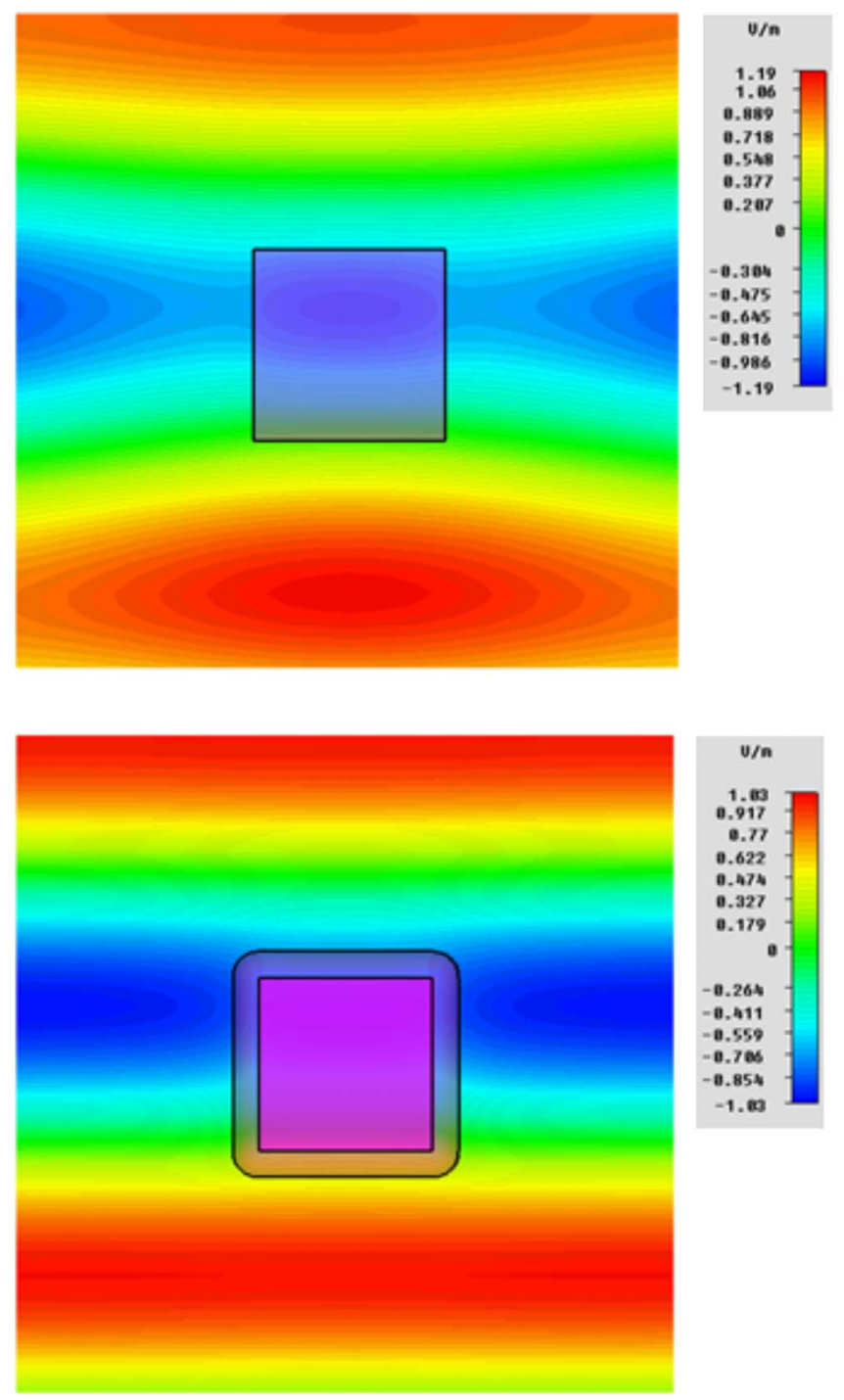
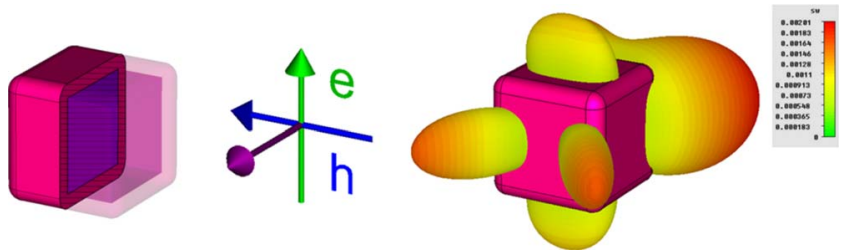

FIG. 14. (Color online) Radar cross-section of a cloaked dielectric cube of silica for the given polarization.

\section{DESIGN OF COMPLEX-SHAPED PLASMONIC COVERS}

We consider in this section different conformal cloaks for dielectric (e.g., made of silica) and plasmonic (e.g., made of silver) objects of different shapes, properly optimized to provide strong scattering reduction for all incidence angles, despite the anisotropic scattering from the object to be cloaked. The plasmonic covers have been modeled as Drude disper-
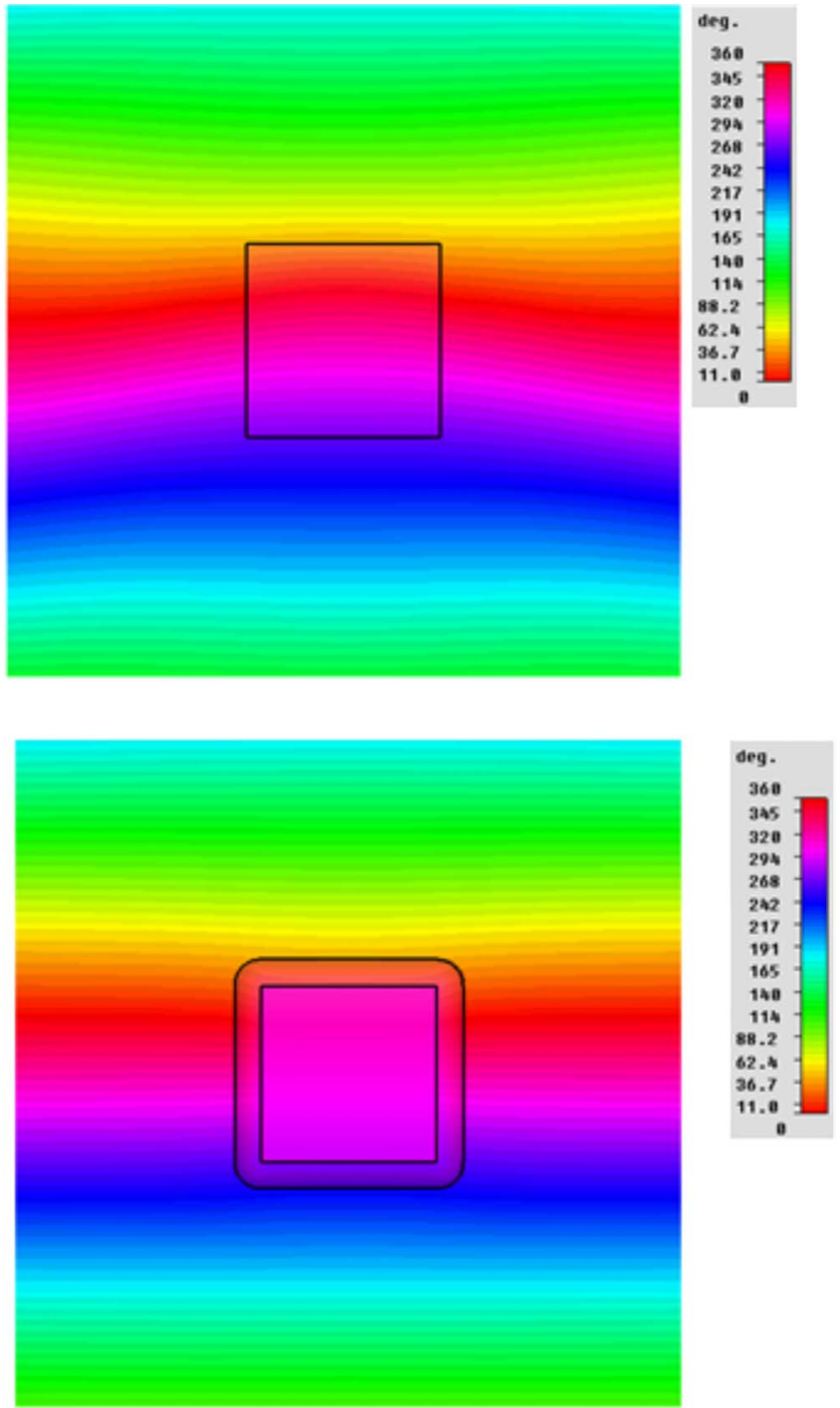

FIG. 15. (Color online) 2D maps of the electric field amplitude (left) and phase (right) for the bare silica cube (top) and the cloaked one (bottom). 

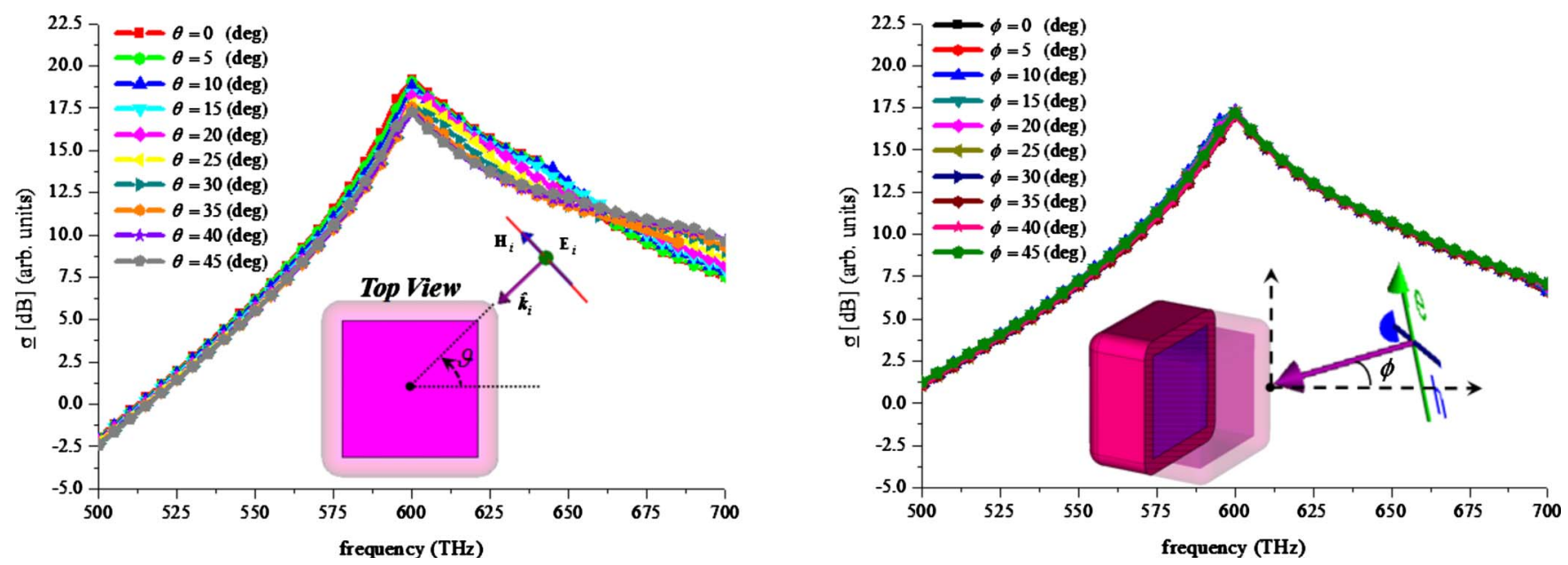

FIG. 16. (Color online) Ratio $\underline{\sigma}$ between the SCS of the bare cube and the cloaked one as a function of the incidence angle.
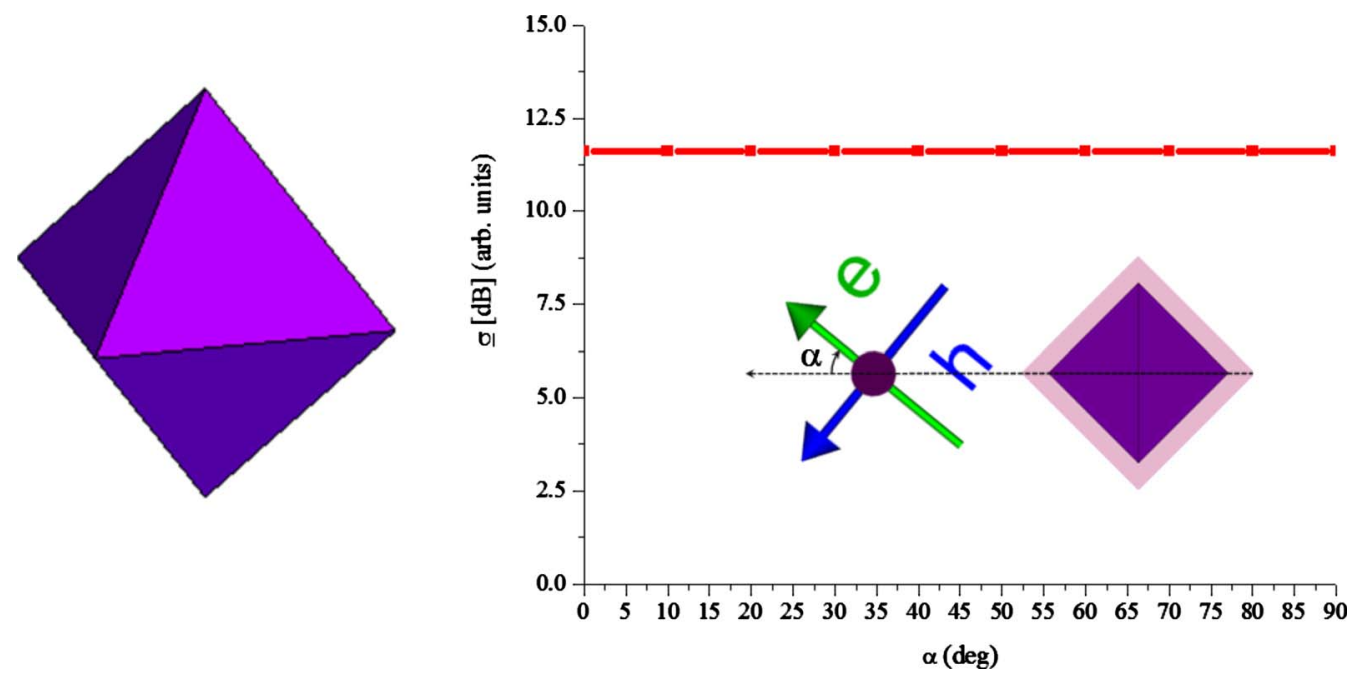

FIG. 17. (Color online) Radar cross-section of a silver octahedron for the given polarization.
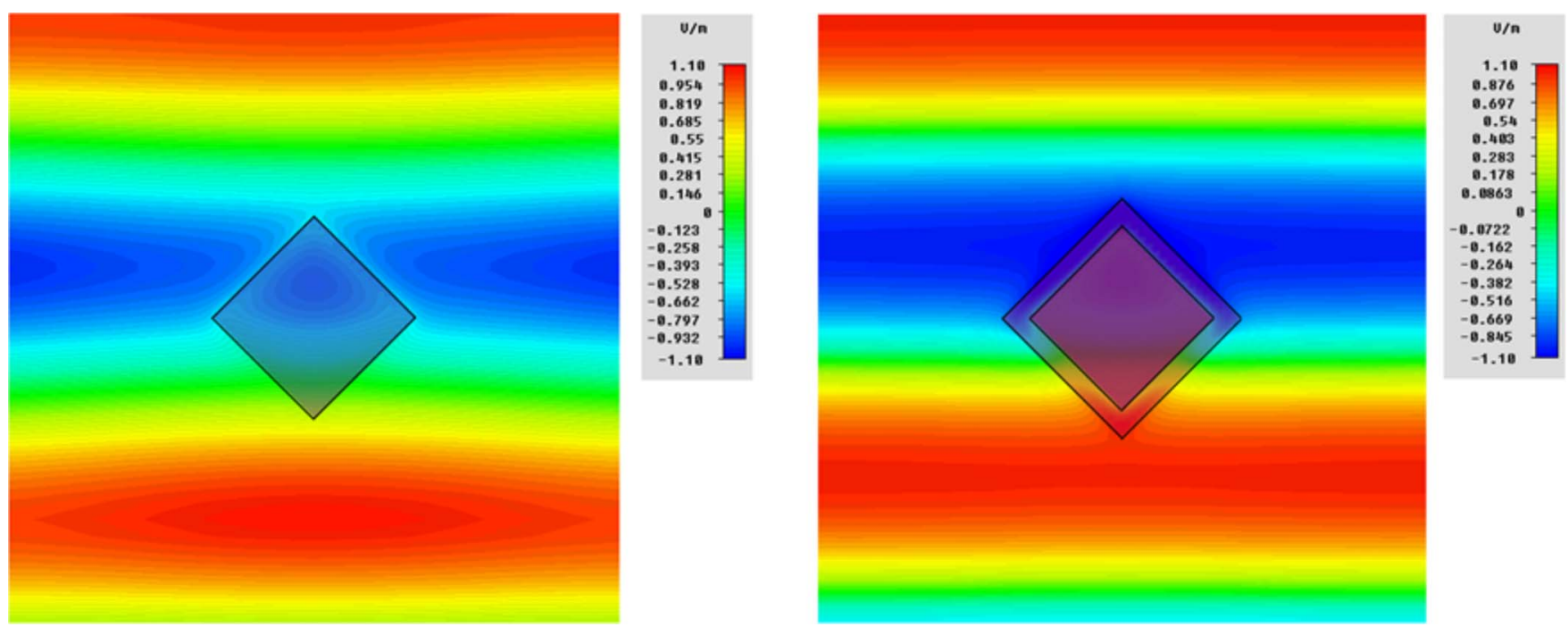

FIG. 18. (Color online) 2D maps of the electric field amplitude for the bare silica octahedron (left) and the cloaked one (right). 

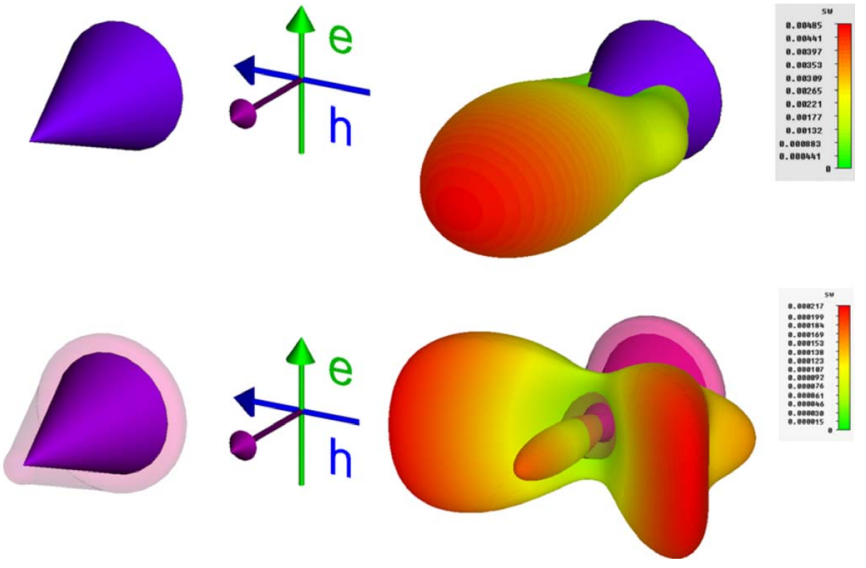

FIG. 19. (Color online) Ratio $\underline{\sigma}$ between the SCS of the silica bare cone and the cloaked one as a function of polarization angle.
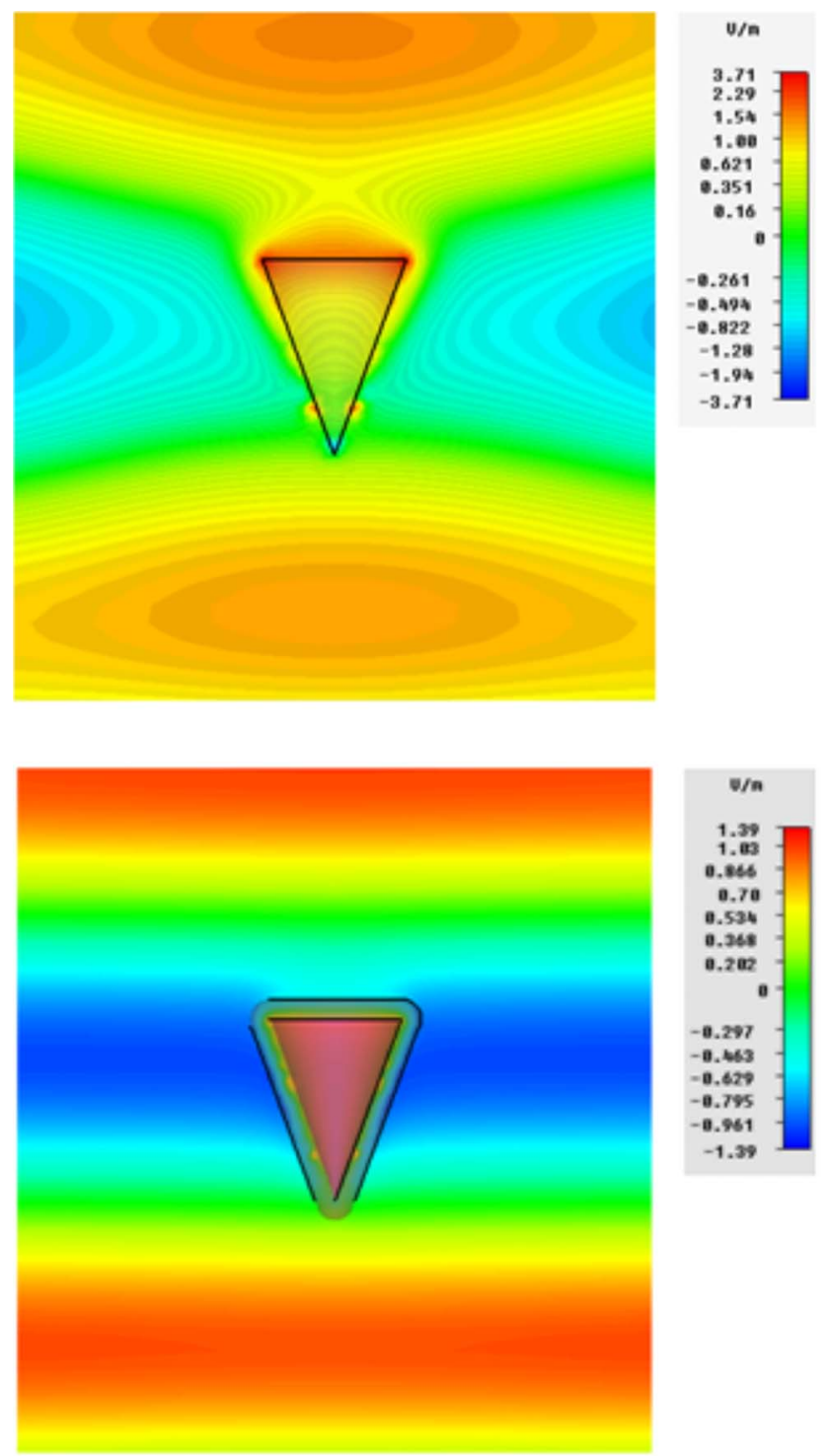

FIG. 21. (Color online) 2D maps of the electric field amplitude (left) and phase (right) for the bare silver cone (top) and the cloaked one (bottom).

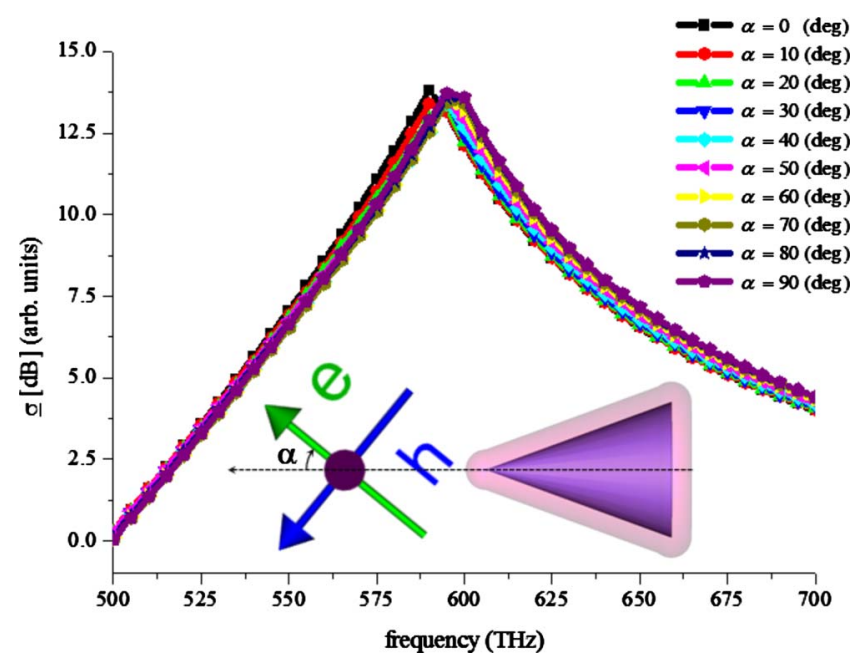

FIG. 20. (Color online) Ratio $\sigma$ between the SCS of the bare cone and the cloaked one as a function of polarization angle.

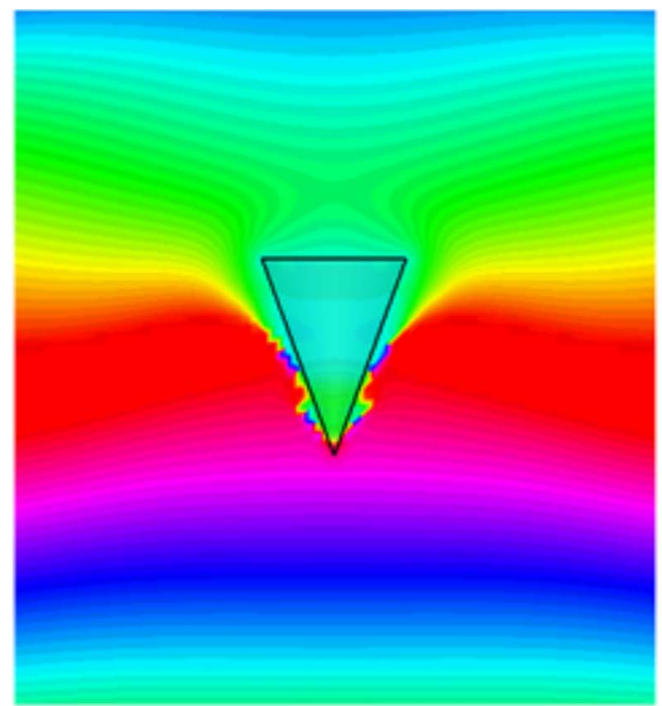

deg.

360
345
320

32

298

242

217

165

140

88.2

62.4

36.7

11.0

.
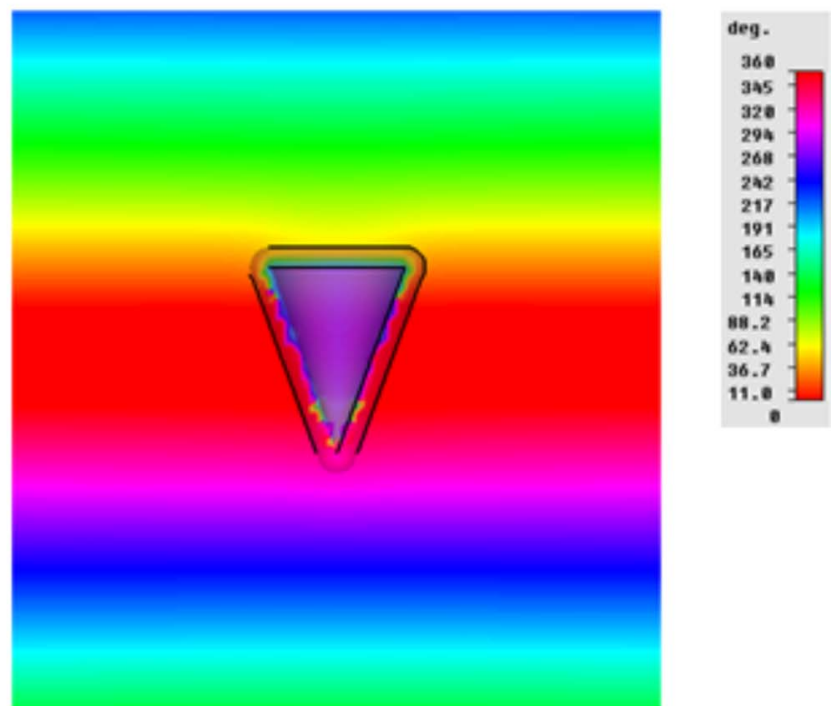

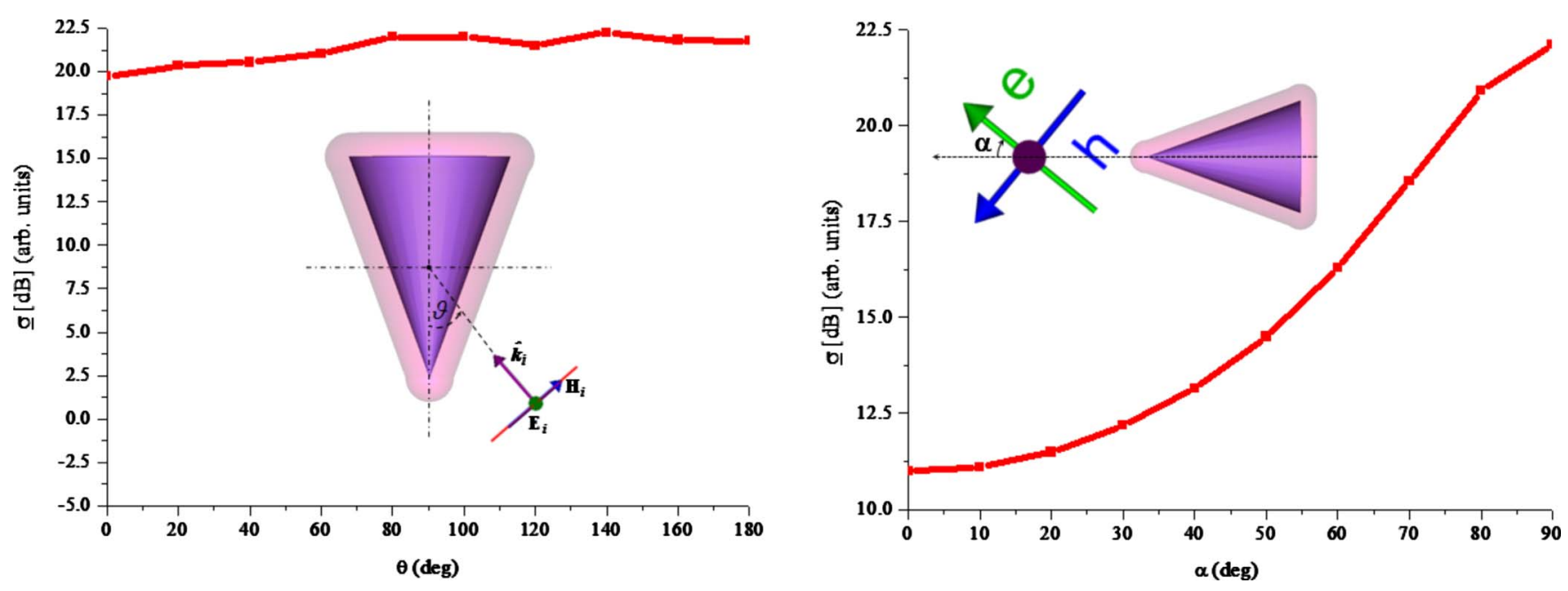

FIG. 22. (Color online) Ratio $\sigma$ between the SCS of the silver bare cone and the cloaked one as a function of the incidence angle.

sive media, which is characterized by the following relative permittivity:

$$
\varepsilon_{r}(\omega)=\varepsilon_{\infty}+\frac{\omega_{p}^{2}}{\omega\left(\omega-j \nu_{c}\right)},
$$

with being $\omega_{p}$ the plasma frequency, $\varepsilon_{\infty}$ as the upper frequency permittivity limit, and $\nu_{c}$ as the damping factor taking into account losses. The characteristic electrical dimension of each obstacle is $200 \mathrm{~nm}=2 / 5 \lambda_{0}$ at the operating frequency of $600 \mathrm{THz}$.

Consider first a dielectric cube of silica [25] with a diagonal length $\ell=280 \mathrm{~nm}$, as in Fig. 13, which reports also the scattering width of the object (measured in squared wavelengths). It is evident that for this size the scattering is not purely dipolar any longer, and higher-order multipoles contribute to a more directive scattered shadow. A proper cloak may be synthesized as a conformal cover, as discussed in the previous section. We have derived with numerical optimization the proper Drude parameters in Eq. (4) to achieve a good scattering reduction with a thickness $d=40 \mathrm{~nm}$. The damping frequency has been assumed $\nu_{c}=10^{-2} \omega_{p}$, which is a realistic value for plasmonic materials at optical frequencies. For a value $\varepsilon_{r} \approx 0.38$ at the design frequency, the scattering is sensibly reduced, as evident in Figs. 14 and 15 (please note the difference in scale between Figs. 13 and 14). In particular, Fig. 15 reports the field distributions in the cloaked and uncloaked case for plane wave incidence from bottom to top of the figure (toward a side of the cube).

Even by changing the angle of incidence for the impinging plane wave we still verify good performances, even in the worst case scenario of incidence at the edge, as shown in Fig. 16. The proper shape of the cloak, designed as outlined in the previous section, produces a quasi-isotropic cloaking effect.

Next, we have simulated a silica octahedron with the same volume as the previous cube, as in Fig. 17. Again performing a numerical optimization, we have found that for $\varepsilon_{r} \approx 0.35$ and a cover thickness $d=26 \mathrm{~nm}$ the SCS may be reduced by almost $12 \mathrm{~dB}$. This value is almost constant with respect to a variation of the angle formed by the electric field vector and one of the principle diagonals of the octahedron, as shown in Fig. 17.

The transparency effect is indeed evident in the 2D maps of the electric field in Fig. 18, where the field lines of the bare silica octahedron and the cloaked one are compared.

The same results may be obtained also for more anisotropic shapes. We have considered, for example, a silica cone with a base diameter of $120 \mathrm{~nm}$ and a height of $160 \mathrm{~nm}$ Fig. 19. For a cover thickness $d=20 \mathrm{~nm}$ and $\varepsilon_{r} \approx 0.46$ we get a very strong reduction of the scattering width, as shown in Fig. 20. Once again, the anisotropic scattering effect of an asymmetric shape such as a cone may be compensated by properly covering the cone with a conformal plasmonic cloak, which presents similar anisotropic response, ensuring that the cancellation mechanism is quasi-isotropic. Clearly, if one had chosen to cloak the cone with a spherical plasmonic cloak, the scattering reduction could have worked only for a limited range of illumination angles due to the strong anisotropy in its shape.

As it can be clearly seen in Fig. 20, instead this structure is not sensitive to variation of the illuminating radiation polarization. It is seen that for electric field parallel to the axis of the cone, stronger polarization is expected, and therefore a larger effective thickness of the plasmonic material has been chosen along the axis. This ensures that the scattering reduction is uniform with the orientation of the electric field, different from the geometry analyzed in [15].

We have also investigated the response of a cone geometrically identical to the one of Fig. 19 and 20, but made of

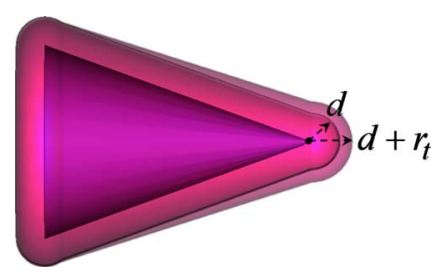

FIG. 23. (Color online) Cloaking cover for the silver cone locally thicker around the tip. 

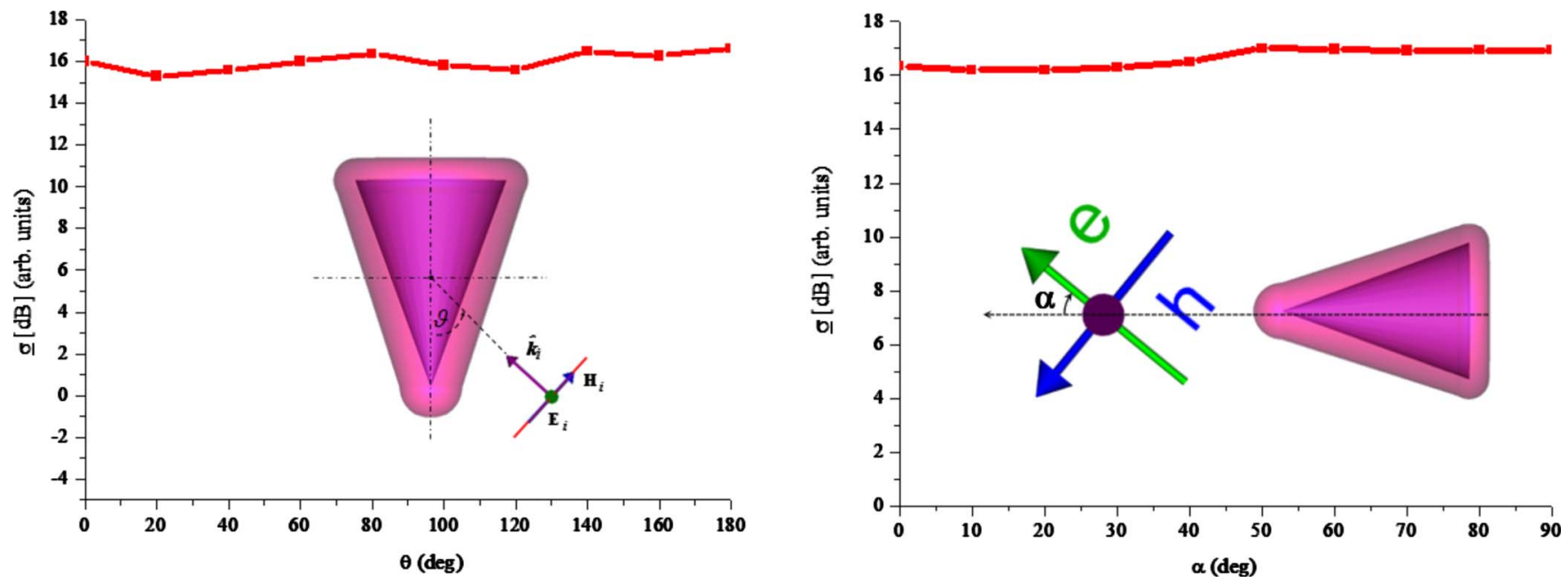

FIG. 24. (Color online) Ratio $\underline{\sigma}$ between the SCS of the silver bare cone and the cloaked one in the new configuration.

silver, with similar results (Fig. 21). Also in this case, despite the less penetrable nature of the conical object, drastic scattering suppression is achieved for $d=20 \mathrm{~nm}$ and $\varepsilon_{r} \approx 0.18$. In this scenario, however, when the electric field lies along the cone axis, the scattering reduction is less isotropic and poorer performance is obtained, due to the inherent electric field enhancement at the plasmonic tip (Fig. 22).

This kind of response is expectable, and is inherently related to the initial choice we adopted to design a conformal cover with a constant thickness. However, for anisotropic scatterers with plasmonic properties, the drastic field enhancement at the tip should be properly taken into account by increasing the cloak thickness near the tip, as shown in Fig. 23. This would ensure, for the same homogeneous material composing the cloak, a larger local polarizability that may compensate the increased scattering from the tip.

By numerically optimizing the cover thickness $d$ near the tip, we obtain for a slight variation $r_{t}=7 \mathrm{~nm}$ of the initial setup an almost isotropic response for all the incidence angles, as reported in Fig. 24.

\section{CONCLUSIONS}

We have extended here the scattering cancellation technique to the case of arbitrarily shaped objects with anisotropic scattering. We have shown how homogeneous covers may be successfully used to cloak moderately sized objects, even for sizes for which the dipolar scattering is not necessarily dominant. Our findings show that a conformal shape is the ideal solution to achieve weak dependence of the scattering reduction upon the polarization of the impinging illumination. Our results ensure that the plasmonic cloaking technique may be applied also to strongly anisotropic dielectric, plasmonic or conducting objects, by properly tailoring the shape and design of the cloaking plasmonic layer.
[1] U. Leonhardt, Science 312, 1777 (2006).

[2] J. B. Pendry, D. Schurig, and D. R. Smith, Science 312, 1780 (2006).

[3] D. Schurig et al., Science 314, 977 (2006).

[4] W. X. Jiang, T. J. Cui, X. M. Yang, Q. Cheng, R. Liu, and D. R. Smith, Appl. Phys. Lett. 93, 194102 (2008).

[5] S. A. Cummer, B.-I. Popa, D. Schurig, D. R. Smith, and J. B. Pendry, Phys. Rev. E 74, 036621 (2006).

[6] A. Alù and N. Engheta, Phys. Rev. E 72, 016623 (2005).

[7] A. Alù and N. Engheta, Opt. Express 15, 3318 (2007).

[8] A. Alù and N. Engheta, Opt. Express 15, 7578 (2007).

[9] A. Alù and N. Engheta, J. Opt. A, Pure Appl. Opt. 10, 093002 (2008).

[10] M. G. Silveirinha, A. Alù, and N. Engheta, Phys. Rev. E 75, 036603 (2007).

[11] F. Bilotti, S. Tricarico, and L. Vegni, New J. Phys. 10, 115035 (2008).

[12] S. Tricarico, F. Bilotti, and L. Vegni, J. Eur. Opt. Soc. Rapid
Publ. 4, 09021 (2009).

[13] F. Bilotti, S. Tricarico, and L. Vegni, IEEE Trans. Nanotechnol. 9, 55 (2009).

[14] M. G. Silveirinha, A. Alù, and N. Engheta, Phys. Rev. B 78, 075107 (2008).

[15] A. Alù and N. Engheta, Phys. Rev. Lett. 100, 113901 (2008).

[16] N. A. Nicorovici, R. C. McPhedran, and G. W. Milton, Phys. Rev. B 49, 8479 (1994).

[17] G. W. Milton and N.-A. P. Nicorovici, Proc. R. Soc. London, Ser. A 462, 3027 (2006).

[18] P. Alitalo, O. Luukkonen, L. Jylhä, J. Venermo, and S. A. Tretyakov, IEEE Trans. Antennas Propag. 56, 416 (2008).

[19] Y. Luo, J. Zhang, B. I. Wu, and H. Chen, Phys. Rev. B 78, 125108 (2008).

[20] W. X. Jiang, T. J. Cui, G. X. Yu, X. Q. Lin, Q. Cheng, and J. Y. Chin, J. Phys. D 41, 085504 (2008).

[21] W. X. Jiang, J. Y. Chin, Z. Li, Q. Cheng, R. Liu, and T. J. Cui, Phys. Rev. E 77, 066607 (2008). 
[22] A. Nicolet, F. Zolla, and S. Guenneau, Opt. Lett. 44, 1150 (2008).

[23] G. Dupont, S. Guenneau, S. Enoch, G. Demesy, A. Nicolet, F. Zolla, and A. Diatta, Opt. Express 17, 22603 (2009).

[24] A. Diatta, S. Guenneau, A. Nicolet, and F. Zolla, Opt. Express 17, 13389 (2009).

[25] A. Noor and Z. Hu, IET Proc. Microwaves, Antennas Propag. 3, 40 (2009).

[26] A. H. Sihvola, Electromagnetic Mixing Formulas and Applications (IEE Press, London, 1999).

[27] J. Avelin, R. Sharma, I. Häninnen, and A. H. Sihvola, IEEE
Trans. Antennas Propag. 49, 451 (2001).

[28] C.S.T. Design Studio ${ }^{\mathrm{TM}}, 2009$, www.cst.com

[29] J. D. Jackson, Classical Electrodynamics (John Wiley \& Sons Inc., New York, 1998).

[30] J. Avelin and A. H. Sihvola, Microwave Opt. Technol. Lett. 32, 60 (2002).

[31] D. F. Herrick and T. B. A. Senior, Appl. Phys. A: Mater. Sci. Process. 13, 175 (1977).

[32] E. Palik, Handbook Of Optical Constants Of Solids (Academic, New York, 1998). 